\title{
Artículos
}

\section{El rostro de la mujer minera en Colombia: un análisis a partir del enfoque de género*}

Cómo citar este artículo: Cifuentes Guerrero, J. A., y Güiza Suárez, L. (202I). El rostro de la mujer minera en Colombia: un análisis a partir del enfoque de género. Cuadernos de Desarrollo Rural, i8. https://doi.org/ı0.III44/Javeriana.cdri8.rmmc

\author{
Julie Alejandra Cifuentes Guerrero ${ }^{a}$ \\ Universidad del Rosario, Colombia \\ julie.cifuentes@urosario.edu.co \\ ORCID: https://orcid.org/0000-0002-8299-7697 \\ Leonardo Güza Suárez \\ Universidad del Rosario, Colombia \\ ORCID: https://orcid.org/0000-0003-0742-3930 \\ DOI: https://doi.org/I0.III44/Javeriana.cdri8.rmmc \\ Recibido: 20 Agosto 2019 I Aceptado: 05 Febrero 202I I Publicación: 25 Agosto 202I
}

\section{Resumen:}

En la minería se presentan enormes desigualdades de género. A partir de este contexto, este artículo presenta un análisis de los aspectos socioeconómicos, culturales y jurídicos de las mujeres mineras en Colombia, con el fin de demostrar la importancia de desarrollar políticas públicas con enfoque de género en el sector minero. Para lo anterior, a partir de un enfoque cualitativo, se revisó sistemática y críticamente la literatura disponible sobre el tema, de la cual se puede concluir que la participación de la mujer en la minería en Colombia se diferencia según la cultura. Así mismo, se descubrió que la mayor fuerza laboral está concentrada en la pequeña minería de oro y en su entorno se presentan situaciones que vulneran los derechos humanos de las mujeres.

Palabras clave: desigualdad de género, derechos humanos, minería, mujer.

\section{The Face of Miner Women in Colombia: An Analysis under a Gender Approach}

\footnotetext{
a Autora de correspondencia. Correo electrónico: julie.cifuentes@urosario.edu.co
} 


\begin{abstract}
:
Mining has huge gender inequalities. In this context, this article presents an analysis of the socioeconomic, cultural and legal aspects of the miner women in Colombia in order to show how important it is to develop public policies under a gender approach for the mining industry. Using a qualitative methodology, a systematic and critical review was done to the literature available on this topic. It is concluded that the woman's participation in the mining industry in Colombia is differentiated based on the culture. In addition, it was found that the bigger workforce is concentrated in the small business of gold mining but many situations occur when the woman's Human Rights are violated.
\end{abstract}

Keywords: gender inequality, Human Rights, mining, women.

Históricamente, la minería ha sido un trabajo ejercido por hombres (Bermúdez et al., 20II), sin embargo, en los últimos años, las mujeres han tenido una mayor participación en la extracción de minerales, principalmente por razones sociales y culturales. En estas, se destaca la falta de oportunidades laborales, lo que las impulsa a ejercer la minería como medio de sustento (Alonso y Rivera Guzmán, 20I8) y, a nivel cultural, para algunas comunidades ser mujer minera es un oficio ancestral que forma parte de su identidad (Carabalí-Viveros y Ladino-Mosquera, 20I4).

En Colombia, la participación de la mujer en la minería se presenta de manera diferenciada, dado el carácter pluriétnico y multicultural del territorio. En departamentos como Antioquia y Santander, históricamente las mujeres son chatarreras y trabajan al escarbar entre los restos de las minas donde laboran principalmente hombres (ONU Mujeres, 20I6). Allí, las mujeres mineras adquieren un gran liderazgo y protagonismo, al consolidarse en asociaciones y cooperativas que les permiten materializar el derecho al trabajo en las labores mineras (Quiroga, 20I4). En contraste, en departamentos como Cauca y Chocó, esta actividad se desarrolla ancestralmente por comunidades afrodescendientes, en las que el cargo de la mujer es diferente al que ocupa en comunidades no minoritarias, pues participa de manera activa en la defensa de su identidad cultural y ejerce la actividad de la minería al mismo nivel que los hombres (Davis, 2013) ; aquí, se desempeñan como barequeras. Entonces, la minería cobra especial protagonismo, al otorgarles la autonomía para su subsistencia (Güiza, 2013), lo que hace que esta actividad se consolide como un aspecto fundamental en las dinámicas económicas y sociales de apropiación del territorio.

Pese a lo anterior, en Colombia, el análisis de la realidad socioeconómica y cultural, así como de los riesgos en materia de derechos humanos de las mujeres mineras, es incipiente. Sobre este asunto, Ulloa (2016) menciona que el enfoque de género no ha sido un tema central en las investigaciones sobre minería, a pesar de que es la actividad extractiva en la que se presentan más desigualdades de género, socioambientales, económicas, laborales y políticas. En su realización se presentan episodios violentos contra las mujeres (Ulloa, 20r6), lo que tiene como resultado que ellas cambien su medios de vida y las formas tradicionales de apropiación del territorio.

Con este escenario, la investigación que aquí se presenta tuvo como objetivo analizar los aspectos sociales, económicos, culturales y jurídicos de las mujeres que realizan actividades mineras de subsistencia y de pequeña escala en entornos rurales, a partir de la perspectiva del enfoque de género. El presente texto tiene como objetivo contribuir a una realidad poco estudiada en el país y así identificar las situaciones de desigualdad a las que dichas mujeres se encuentran expuestas. El desarrollo conceptual de este artículo inicia con la presentación de algunas consideraciones de género en la minera y la exposición de algunas generalidades sobre la participación de la mujer en esta actividad en Colombia; la clasificación de dichos aspectos se realizó en razón a criterios multiculturales y al desarrollo jurídico del Estado colombiano. En los siguientes apartes, se analizan y discuten las condiciones socioeconómicas de la vida de las mujeres mineras, en las que se incluyen aspectos laborales, familiares y de salud, así como algunas de las implicaciones de la minería ilegal, relacionadas con la vulneración de sus derechos humanos. La investigación finaliza con la presentación de las conclusiones y las recomendaciones producto de esta. 


\section{Método}

Este artículo es el resultado de una investigación cualitativa, crítica y analítica, realizada a partir de la aplicación de la técnica de análisis documental, con el fin de analizar la participación de la mujer en la minera en los ámbitos social, económico, cultural, ambiental y jurídico. La investigación se elaboró por medio de reflexiones teóricas en las que se abordó el tema de manera deductiva, es decir, con la descripción de las variables antes mencionadas en un contexto general, para luego analizarlas en casos particulares. Así, se trabajó la minería de subsistencia y de pequeña escala, realizada en contextos rurales por comunidades campesinas, indígenas, afrocolombianas y no minoritarias. De esta manera, se propusieron elementos útiles para la formulación de políticas públicas en esta materia.

\section{Desarrollo del tema}

\section{Consideraciones de género en la minería}

A lo largo de la historia, han existido discursos de género que limitan la participación de las mujeres en los diferentes escenarios de toma de decisiones, participación política, desempeño laboral, entre otros (Bermúdez et al., 20II). En la minería, este paradigma está relacionado con lo que cotidianamente se ha establecido como adecuado para lo "femenino" y lo "masculino", el cual refuerza las ideas de que las mujeres están en la obligación de velar por el bienestar de la familia, son delicadas, frágiles, sensibles y débiles. Mientras tanto, el papel de los hombres se relaciona con la fuerza bruta y la libertad en el acceso al mundo público y a la recreación (Jiménez et al., 20I4).

Bermúdez et al. (2014) mencionan que "la construcción social de género ha estado articulada históricamente con una desigual relación de poder entre hombres y mujeres, que a su vez se ha expresado en una desigual distribución de derechos, propiedades y responsabilidades” ( p. 19). Esta afirmación resulta crucial para el análisis del papel de la mujer en la minería en Colombia, ya que los antecedentes de participación de aquella en dicha actividad se relacionan con la existencia de prejuicios y cuestionamientos sobre su capacidad física e intelectual para el desarrollo de las funciones. La minería es una actividad que se caracteriza por ser eminentemente machista, pues existe una marcada disputa de género, que obliga a las mujeres a adecuarse a estereotipos y ganarse el respeto de los hombres para operar en las minas. En este contexto, ser mujer minera significa superar aquel lugar de desventaja en el cual están situadas, como resultado de los discursos y significados dominantes de la jerarquía de género, impuestos por la sociedad y la cultura. Por este motivo, las mujeres deben reconstruir su dignidad a partir de un comportamiento que responda al carácter de reconocimiento social que buscan los individuos en un mundo cada vez más competitivo y con menos oportunidades. Para las mujeres, ocupar un lugar típicamente masculino produce un nivel de importancia que les posibilita tener voz (Soraire et al., 20I3), así como luchar por la igualdad laboral y una mejor calidad de vida.

A nivel de políticas públicas, las consideraciones de género en la industria minera han tenido poco impacto en la incorporación de mujeres a este oficio. Así lo muestra la distribución laboral de la minería en Colombia, en la que se ve que la mayor oportunidad de empleo para las mujeres está en la minería informal (Güiza, 2013), principalmente en actividades operativas poco remuneradas.

Este comportamiento ha estado determinado por falencias en la identificación de las mujeres como un grupo diferenciado de actores en la planificación y operación de las minas, pues está demostrado que la consideración 
del enfoque de género trae beneficios para las empresas mineras. Un ejemplo de lo anterior es la compañía minera de origen chileno Gabriela Mistral, que posee el mayor porcentaje de participación femenina en la minería en este país, con un total del $26 \%$ de las plazas en áreas productivas y administrativas ocupadas por mujeres. Con la entrada de trabajadoras a esta compañía, se redujeron los índices de accidentalidad y ausentismo, y hubo mejoras significativas en el clima laboral y en la productividad (Codelco, 2013) .

En este sentido, la minería puede considerarse como una actividad económica más en la que las mujeres tienen las mismas posibilidades de desarrollo que los hombres, en relación con su capacidad física e intelectual. De los argumentos a favor de la inclusión de las mujeres en la minería se destacan: el alto grado de compromiso en el logro de objetivos, su capacidad de manejo de múltiples variables de forma simultánea, la flexibilidad para adecuarse a los cambios, la disciplina para actuar ordenadamente, su intuición y percepción, la facilidad para seguir los pasos observo, pienso y actúo (Soto, 20II), una mayor responsabilidad (Jiménez et al., 20I4), y su condición física-natural de delicadeza, que representa un valor agregado en el manejo de maquinaria y personal (Soraire et al., 2013).

Finalmente, en el marco de la Agenda 2030 para el Desarrollo Sostenible (ONU, 2015), aprobada por Colombia a través del Conpes 39ı/20I8, considerar el enfoque de género a nivel laboral y en la distribución de la riqueza permite avanzar hacia una sociedad más igualitaria. Esto representa un gran reto para los países, pues, como lo afirma el Foro Económico Mundial (World Economic Forum, 2018), con el ritmo actual de progreso tomará doscientos dos años cerrar la brecha salarial entre hombres y mujeres.

\section{Participación de la mujer en la minería}

Históricamente, la participación de las mujeres en la minería ha estado limitada por aspectos culturales y sociales. Por ejemplo, la creencia popular de la mala suerte que traen las mujeres sobre la producción minera, la condición consustancial comúnmente aceptada de la mujer como responsable de las labores domésticas y de crianza, la idea de la debilidad del género femenino (Soto, 20II), y las situaciones de conflictividad que generan las mujeres en el ámbito laboral minero.

En América Latina, la inserción laboral femenina en la minería esta permeada por este tipo de mitos y creencias que han influenciado la disposición de los hombres mineros para aceptar mujeres como compañeras de trabajo (Jiménez et al., 20I4). En Bolivia, por ejemplo, existe un mito relacionado con la mala suerte que trae la presencia de mujeres en las minas. Entre los trabajadores, se creía que en los socavones vivían el Tío y la Pachamama. El primero es el patrón de las vetas y del trabajo minero y está en deuda con los hombres que lo alimentan, por lo que revela a los mineros los yacimientos a cambio de ofrendas. La segunda es la deidad femenina, con quien se comparte hoja de coca, alcohol y comida para agradecer lo recibido o pedir por lo deseado (Soraire et al., 20I3). Influenciados por esta creencia, los mineros sentían temor del ingreso de mujeres a las minas, pues se pensaba que la Pachamama se pondría celosa y el Tío escondería las vetas. Por esta razón, las mujeres solo tenían permitido buscar restos en las laderas de los cerros (Viezzer, 2005). Esta creencia, como se explicará más adelante, ha influenciado la modalidad de trabajo actual de la mujer minera en Bolivia.

Cabe resaltar que estos prejuicios han sido reemplazados por otros como la incompatibilidad de la minería con las funciones de las mujeres como madres, la imposibilidad de realizar labores que impliquen fuerza y el aumento en los costos que genera contar con mujeres en las empresas mineras, por aspectos como licencias de maternidad y horarios de lactancia (Stefanovic y Saavedra Alvayay, 20I6).

En el caso de Colombia, Carabalí-Viveros y Ladino-Mosquera (20I4) mencionan que "no puede verse la presencia femenina en las minas como un hecho aislado de la realidad del país, pues dicha actividad se enmarca en la historia extractiva de Colombia” (p. 265). Esta afirmación es compartida por Ramos (20II), al establecer que la participación de la mujer en la minería data de "la época de la Colonia donde existían grupos de hombres y 
mujeres quiénes a través de la técnica de aluvión extraían alrededor del 95\% del oro del Virreinato de la Nueva Granada” (p. 15). Sin embargo, se trata de una inclusión desigual que no reconoce los derechos laborales y humanos de las mujeres mineras (Ulloa, 20I4).

Según cifras del censo minero 2010-20II (Ministerio de Minas y Energía, 20I2), la minería (legal e ilegal) genera

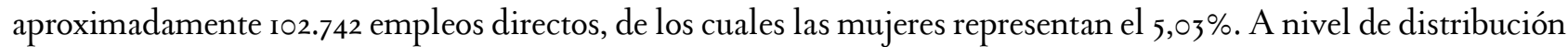
laboral, las mujeres realizan principalmente actividades operativas (con una participación del 47,3\%), seguidas de cargos administrativos (con el 45,I\%) y de puestos ejecutivos (el 7,51\%). En cuanto a la extracción de minerales, la del oro es en la que hay mayor participación de mujeres (el $45 \%$ de la unidad de producción minera (UPM) de oro, con al menos una mujer que labora), seguida por la extracción de arena (I4,I7\%), arcilla (II,43\%) y carbón (II,40\%) (Ministerio de Minas y Energía, 20II).

La minería de subsistencia y de pequeña escala, que no fue considerada en el censo minero de 20II, es una actividad legal y se realiza en su totalidad en contextos rurales. Para su desarrollo no se requiere contar con título minero, siempre que se haga de manera artesanal; la persona solo se debe inscribir en el Registro Único de Comercializadores de Minerales (Rucom), según el cual, para agosto de 2019, había 81.482 barequeros (45\% mujeres y $55 \%$ hombres) y 5432 chatarreros ( $43 \%$ mujeres y $57 \%$ hombres). El departamento de Antioquía es el que cuenta con mayor presencia de mujeres que realizan labores mineras, con el 90,2\%; del 9,8\% restante, Cauca concentra en $5,5 \%$, y el resto se distribuye en departamentos como Boyacá. Vale la pena señalar que, con relación a las mujeres que desarrollan el barequeo, en los departamentos de Nariño, Valle, Chocó y Cauca, estas superan en porcentaje a los hombres, con una proporción del $55 \%$ en Nariño y Valle, 54\% en el Chocó y $51 \%$ en el Cauca (Agencia Nacional de Minería [ANM], 2019.

Como puede apreciarse, y en línea con lo que afirma Güiza (2013), en el país la demanda de trabajo de la mujer en la minería se da a nivel informal y en su totalidad en contextos rurales, en los que persiste la desigualdad de género que lleva a que se acentúen situaciones de inequidad laboral.

De manera indirecta, la actividad minera genera oportunidades de empleo para mujeres en la prestación de servicios como alimentación, hospedaje y lavado de ropa, para los hombres que trabajan en las minas; en muchos casos, estas labores representan una importante fuente de ingresos (Chaparro y Lardé, 2009). Como se analizará más adelante, la prostitución también se posiciona como una fuente de trabajo indirecta para las mujeres en los entornos mineros, la cual está cobrando un fuerte protagonismo como una forma de subsistencia (Rodríguez, 20I3). Dada la condición pluriétnica y multicultural del país, la participación de la mujer en la minería es diferenciada y está condicionada por las diversas formas de apropiación cultural del territorio, tal y como se describe a continuación.

\section{Comunidades campesinas}

En departamentos como Boyacá, tradicionalmente de vocación agrícola y territorialidad campesina, las mujeres, impulsadas por la falta de oportunidades laborales y la baja remuneración económica -que en muchas ocasiones no supera los $\$ 200.000$ al mes (ONU Mujeres, 20I6) -, han optado por la minería informal de esmeraldas y la extracción artesanal de arcilla como parte de su economía de subsistencia. En Ráquira, por ejemplo, las mujeres que extraen arcilla producen artesanías como una alternativa de sustento, lo que les facilita permanecer cerca de sus hogares y cuidar de sus hijos (Centro Regional de Empresas y Emprendimientos Responsables de Colombia [Creer], 20I6). En municipios como Muzo, Maripí, Santa Barbará, Otanche y San Pablo de Borbur, desde hace más de sesenta años, las mujeres se desempeñan como "guaqueras" que, motivadas por la falta de oportunidades laborales y la situación de desamparo, al perder a sus maridos durante la guerra del Oro Verde, ven en la recuperación de esmeraldas de las minas el único sustento para sus familias. A nivel de organización social, en Muzo, las guaqueras crearon la Fundación de Mujeres con Propósito por Boyacá, con el objetivo de buscar 
financiación para el desarrollo de proyectos agrícolas, avícolas y textiles, pues la obtención de esmeraldas es cada vez más escaza (ONU Mujeres, 20I6) .

\section{Comunidades afrocolombianas}

Ancestralmente, las comunidades afrocolombianas han desarrollado actividades mineras, desde mediados del siglo XVII, como una forma cultural de apropiación del territorio. Según Bolívar Rocha e Ibarra Melo (2017) “desde el tiempo de la esclavitud, se han dedicado a las actividades mineras y agrícolas como sustento, no solo económico, sino también social y cultural” (p. 163). En palabras de Bernasconi (2014), las actividades mineras para estas comunidades son herramientas de lucha y resistencia en defensa del territorio heredado, que le permiten a la comunidad permanecer en el tiempo y conservar su identidad, tradición y forma de organización social.

La minería de subsistencia y de pequeña escala de oro ha estado presente en muchas de las regiones del Cauca y el Pacífico desde la Colonia (Mina et al., 2015). En el departamento del Chocó, por ejemplo, el 60,1\% del total de las mujeres que desempeñan labores mineras lo hacen en la UPM de oro (Ministerio de Minas y Energía, 20II). Para las mujeres afrocolombianas, la minería hace parte de sus vidas desde la niñez, pues, como lo afirman mujeres mineras de Suárez (Cauca), a través de los procesos de crianza, los padres, las madres y las abuelas trasmiten el conocimiento minero, tanto a hombres como a mujeres. En territorios afrocolombianos, las mujeres ejercen la minería con libertad, lo que les permite organizarse de acuerdo con sus formas de trabajo y tiempo, y así armonizar el oficio de la minería con la agricultura y el cuidado del hogar (Carabalí-Viveros y Ladino-Mosquera, 20r4). Lo anterior obedece a un comportamiento cultural de las comunidades afrocolombianas específicamente, pues estas son matrifocales, lo que, según Posso Quiceno (2010), quiere decir que el orden del hogar gira en torno a la mujer.

Las mujeres afrocolombianas que se dedican a la minería de subsistencia y de pequeña escala de oro lo hacen a través del barequeo o mazamorreo (Creer, 20I6). Esta modalidad de la minería se realiza de forma manual y a las orillas de los ríos. Las mujeres mueven con precisión la batea de un lado para decantar las partículas de tierra, arena y grava más pesadas, para así poder seleccionar el oro (Carabalí-Viveros y Ladino-Mosquera, 20r4).

Las organizaciones de las mujeres mineras afrocolombianas se caracterizan por ser importantes movimientos sociales que luchan y defienden la minería ancestral, frente a la minería formal a gran escala (Blanco Acendra, 2017) y a la minería ilegal. Esto se refleja en el número de organizaciones colectivas lideradas por mujeres, como el Consejo Comunitario de La Toma y la Asociación Municipal de Mujeres de Buenos Aires (ASOM), las cuales les han permitido acceder a la justicia para defender su derecho fundamental a la consulta previa y manifestar su oposición a la presencia de multinacionales mineras en sus territorios, pues consideran que estas vulneran su autonomía, devalúan sus tradiciones y fragmentan el territorio ancestral (Posso Quiceno, 2010).

\section{Comunidades indígenas}

A diferencia de las comunidades afrocolombianas, en las que hombres y mujeres han practicado la minería durante siglos, en territorios indígenas, como los que se encuentran ubicados en la Amazonía colombiana, la inserción de la mujer en la minería es un hecho reciente. Lo anterior se debe a que tradicionalmente en estas comunidades las funciones de los hombres y las mujeres se han asignado de acuerdo con las historias del origen del mundo, en las que la mujer es responsable de la agrobiodiversidad y la seguridad alimentaria (Rodríguez y Rubiano, 20ı6). Pese a lo anterior, en departamentos como Caquetá, Putumayo y Guainía, factores como la pérdida de la economía de sustento, la destrucción de formas tradicionales de vida comunitaria y la presencia de minería ilegal (Moncada, 
2017) impulsan a las mujeres indígenas a involucrarse en la cadena de valor minera con la proporción de alimentos, hospedaje, lavado de ropa y servicios sexuales (Rodríguez y Rubiano, 20I6). Estas labores no son estables y son desarrolladas con la esperanza de garantizar su autonomía y la de sus hogares. Para algunas mujeres indígenas, la prostitución es una actividad considerada como una de las pocas fuentes de ingreso "dignas" (Rodríguez, 20I3).

Con respecto a la organización social, no existen asociaciones, cooperativas o estructuras similares de mujeres mineras que les permitan luchar por sus derechos y acceder a mejores condiciones laborales. Sin embargo, organizaciones no gubernamentales como GAIA Amazonas, la Organización Nacional de Pueblos Indígenas de la Amazonía Colombiana (Opiac), entre otras, han luchado por sus derechos y por la autonomía de las comunidades indígenas que habitan esta región.

\section{Grupos no minoritarios}

$\mathrm{Al}$ igual que ocurre en las comunidades indígenas y campesinas, en los grupos no minoritarios (por ejemplo, en algunos municipios de Antioquia y Nariño) las mujeres ejercen la minería informalmente, motivadas por el alto nivel de desempleo. Ellas prefieren desempeñarse como mineras y no en empleos formales, pues, a pesar de la inestabilidad laboral de la actividad, esta les proporciona libertad de horarios y reglas, lo que les permite cuidar a sus hijos, dado que la gran mayoría son madres cabeza de familia (Creer, 2016).

Este grupo de mujeres lleva a cabo labores de recolección de los residuos minerales de las minas (Quiroga, 20I4), en diferentes modalidades como chatarreras, hueveras o costaleras. Las costaleras se encargan de lavar los costales en los que se almacena la roca que contiene el mineral y de recuperan los residuos. Las hueveras se acercan a los entables mineros y piden balto (residuos), tamizan el material menos puro, lo recogen en baldes y lo dejan en agua, para así poder elegir las mejores fracciones y comercializarlas (Creer, 2016). El chatarreo constituye la modalidad minera más común entre las mujeres de grupos no minoritarios (Alonso y Rivera Guzmán, 2018) y consiste en la recolección y selección de los residuos a las afueras de las minas. Estas actividades, al igual que el barequeo y la guaquería, no son un oficio permanente y dependen de las labores de extracción del mineral, del espesor de la veta, del contenido de mineral en la roca y de la oportunidad que los mineros les proporcionen a las mujeres para recuperar los residuos (Carabalí-Viveros y Ladino-Mosquera, 2014).

A nivel de organización social, las mujeres chatarreras han conformado asociaciones y cooperativas para mejorar sus condiciones laborales (Quiroga, 20I4). Un aspecto particular de las mujeres mineras de este grupo es que han logrado ejercer la minería de subsistencia y de pequeña escala de oro formalmente, como es el caso en el municipio de La Llanada (Nariño), donde funciona una de las cinco minas formalizadas con certificación Fairmined. La inclusión de mujeres mineras a la empresa les ha permitido a aquellas aumentar su nivel de organización, mejorar su seguridad y estabilidad laboral, fortalecer sus capacidades y aumentar su participación en la toma de decisiones, lo que se ha reflejado en un mejor ambiente laboral y en el aumento de la productividad (Alianza por la Minería Responsable, 2019a).

\section{Aspectos jurídicos de la inclusión de la mujer en la minería en Colombia}

En el marco internacional, Colombia ha suscrito una serie de tratados desde i960, en los cuales se compromete a adoptar medidas para la protección de la mujer y la no discriminación, tales como: el Convenio Internacional del 
Trabajo, relativo a la discriminación en materia de empleo y ocupación, adoptado en 1958 y aprobado en Colombia a través de la Ley 22 de 1967; la Convención sobre la Eliminación de Todas las Formas de Discriminación contra la Mujer, que formó parte del ordenamiento jurídico del país a través de la Ley 5i de i98I y el decreto nacional I398 de 1990, y la Convención Belém do Pará para Prevenir, Sancionar y Erradicar la Violencia contra la Mujer, aprobada en la Ley 248 de 1995 .

Pese a lo anterior, el país se ha tardado significativamente en implementar políticas y enfoques de género que den cumplimiento a dichos tratados, pues, como se evidencia en el contexto laboral colombiano, desde i950 ya existía una restricción a la discriminación de la participación de la mujer en la minería. El numeral 3 del artículo 242 del Código Sustantivo del Trabajo establece que: "Las mujeres, sin distinción de edad, y los menores de dieciocho (I8) años no pueden ser empleados en trabajos subterráneos de las minas ni, en general, trabajar en labores peligrosas, insalubres o que requieran grandes esfuerzos” (Presidencia de la República de Colombia, 1959, Art. 242). Es decir que las mujeres solo podían trabajar en cargos de dirección o supervisión, lo que restringía las oportunidades laborales de las barequeras y chatarreras, pues estas, por lo general, tienen un bajo nivel de escolaridad que no les permite acceder a dichos empleos.

Estas disposiciones fueron declaradas inexequibles por la Corte Constitucional solo hasta el año 20I6, a través de la Sentencia C-586, por considerarse contraria a la Constitución y a los derechos de igualdad, trabajo y libertad de escoger profesión u oficio. El máximo tribunal consideró que esta prohibición era discriminatoria al reforzar el estereotipo de la mujer como "sexo débil" con condición de inferioridad. Adicionalmente, este concluyó que las mujeres de dieciocho años están en plena capacidad de hacer uso de su libertad y autonomía para elegir la profesión u oficio que desean ejercer, incluso si con dicha decisión se asumiesen riesgos para la salud y la vida (Corte Constitucional de Colombia, 2016).

Con respecto al salario, en Colombia existe la Ley i496 de 20II, por medio de la cual se garantiza la igualdad salarial entre mujeres y hombres y se establecen mecanismos para erradicar cualquier forma de discriminación. Pese a esto, la distribución del salario en Colombia es bastante desigual: en promedio, las mujeres ganan entre un $20 \%$ y un $40 \%$ menos que los hombres y en los cargos directivos las mujeres ganan solo un $8 \mathrm{r} \%$ de lo que ganan los hombres (González, 2017) . Lo anterior es significativo si se tiene en cuenta que, según la Comisión Económica para América Latina y el Caribe (Cepal) (20I8), Colombia es el segundo país más desigual en la distribución del ingreso de América Latina: el ı\% más rico de la población concentra el 20\% del ingreso.

Solo en los últimos diez años, con la expedición del Plan Nacional de Desarrollo (PND) 2010-20I4, es que Colombia ha empezado a implementar lineamientos tendientes a la aplicabilidad de los tratados internacionales antes mencionados. El PND 2010-20I4 consagró en los artículos i77 y 179 la obligación de construir, bajo la coordinación de la Alta Consejería Presidencial para la Equidad de la Mujer, una Política Nacional Integral de Género que permita garantizar los derechos humanos integrales e interdependientes de las mujeres y la igualdad de género, así como la implementación de acciones para identificar la magnitud del trabajo no remunerado y la discriminación laboral. En línea con lo anterior, en 2012, el Gobierno nacional presentó los lineamientos de la Política Pública Nacional de Equidad de Género, los cuales sirvieron de fundamento para el Decreto 1930 de 2013, en el cual se adopta dicha política y se crea la Comisión Intersectorial para su implementación. En 2018, a través del Conpes 3918, Colombia adopta la Agenda 2030 y sus diecisiete Objetivos de Desarrollo Sostenible (ODS), como elementos integradores de todas las agendas que actualmente adelanta el país en materia de desarrollo. En temas de igualdad de género, el Conpes destaca que

Colombia tiene como reto seguir trabajando para alcanzar la igualdad de género y el empoderamiento de la mujer. Para esto, se requiere concentrar los esfuerzos en el impacto de los derechos humanos de las mujeres, así como seguir trabajando para reducir las desigualdades económicas, sociales y políticas entre hombres y mujeres, con el fin de disminuir las brechas existentes. (Departamento Nacional de Planeación [DNP], 2018, p. 19) 
Recientemente, a través de la resolución 40796 de 20I8, el Gobierno colombiano adoptó la Política en Derechos Humanos del Sector Minero Energético, que tienen como objetivo "generar un marco de acción que permita al sector minero energético fortalecer su contribución en la prevención, promoción, protección y garantía de los derechos humanos, con enfoque diferencial” (Gobierno de Colombia, 20I8. p. I2). La política contempla el desarrollo de acciones específicas para grupos con especial protección constitucional, como las mujeres, de tal forma que se garantice la inclusión del enfoque diferencial y de género en la identificación y definición de actividades a realizar para cada uno de los lineamientos; en estos se encuentran acciones articuladas con organizaciones de mujeres que permiten el goce efectivo de sus derechos (Ministerio de Minas y Energía, 2018) .

Sumado a lo anterior, con el ingreso de Colombia a la Organización para la Cooperación y el Desarrollo Económicos (OCDE), el país asume retos en esta materia, como la incorporación del enfoque de género a la contratación, el desarrollo profesional y los ciclos presupuestarios, a partir de la promoción de un enfoque integral y coordinado para reducir diferencias salariales y la segregación profesional entre hombres y mujeres (OCDE, 2019) . Para avanzar en estos retos, desde 20I4, Colombia se adhirió como candidato a la iniciativa del Extractive Industries Transparency Initiative (EITI), que busca "proveer información veraz, oportuna, contextualizada y socialmente útil para fortalecer la transparencia en la cadena de valor del sector extractivo en beneficio del desarrollo sostenible local y nacional" (EITI Colombia, 2018). Esta iniciativa propone un estándar sobre el cual las empresas extractivas reportan sus prácticas en temas como licencias y contratos, producción, recaudación de impuestos, asignación de ingresos y contribución social y económica, con el fin de lograr una mayor transparencia y rendición de cuentas (EITI, 2016). A partir de marzo de 2019, el estándar incluye disposiciones de género, bajo el fundamento de que "si bien los hombres son quienes principalmente disfrutan los beneficios de los proyectos de las industrias extractivas, las mujeres sufren una parte desproporcionada de los impactos sociales, económicos y ambientales negativos" (EITI Secretariat, 2019).

\section{Discusión}

En esta sección se presentan los resultados obtenidos a partir del análisis crítico y sistemático que se realizó con los casos documentados en Colombia sobre mujeres mineras, en los aspectos socioeconómicos y en las situaciones que pueden vulnerar sus derechos humanos, principalmente en la minería ilegal.

\section{Aspectos socioeconómicos de la mujer minera}

\section{Situación económica y laboral}

Como se ha explicado, el trabajo de las mujeres en las minas es informal y se basa en su mayoría en el rebusque. Teniendo en cuenta esta situación, las mujeres mineras no cuentan con un salario fijo y no tienen acceso a garantías laborales, tales como seguridad social y licencia de maternidad. Sumado a lo anterior, la actividad minera desarrollada por las mujeres se caracteriza por su alto grado de inestabilidad e intensificación laboral (Ulloa, 20I6). En cuanto a este último aspecto, las jornadas laborales de las mujeres mineras presentan diferencias sustanciales frente a las de los hombres (Alianza por la Minería Responsable, 2012), ya que, como se mencionó, históricamente 
las mujeres han tenido la responsabilidad social de crianza, lo que conlleva a que, adicional al trabajo minero, deban encargarse de los cuidados familiares y domésticos.

Estas tareas se conocen como economía del cuidado, que, según la Ley I413 de 2010, "hace referencia al trabajo no remunerado que se realiza en el hogar, relacionado con mantenimiento de la vivienda, los cuidados a otras personas del hogar o la comunidad y el mantenimiento de la fuerza de trabajo remunerado" (Congreso de la República, 20Io, Art. 2). Esta categoría es fundamental en la economía de Colombia, pues esta, en 20I8, se valoró en I85,7 billones de pesos, es decir, el 20\% del PIB del país; es ejercida principalmente por mujeres (Departamento Administrativo Nacional de Estadística [DANE], 2018) y, sin embargo, no es remunerada.

Cuando las mujeres están vinculadas directamente a empresas mineras, los procesos de contratación muchas veces no cuentan con enfoque de género que reconozca sus derechos laborales por embarazos o tiempo de crianza, lo que demuestra que el proceso de inserción laboral está pensado para hombres (Ulloa, 20I6). Según testimonios de empresas mineras, recopilados por el Creer (2016), la contratación de las mujeres es más baja que la de los hombres porque "salen más caras" y "hacen menos". Con respecto a la remuneración económica, esta es significativamente inferior a la recibida por los hombres, además de ser muy inestable, pues algunas veces las mujeres no logran obtener minerales de las vetas o de los restos de los cerros.

En términos de uso del salario, las mujeres mineras distribuyen sus ingresos en el bienestar y la calidad de vida a sus familias, aspecto que, como se analizará a continuación, difiere del comportamiento usual del hombre minero, quien invierte una parte significativa de sus ingresos en el ocio (Chaparro y Lardé, 2009).

\section{Situación familiar}

La mayoría de las mujeres mineras son cabeza de familia, lo que conlleva a que todas las responsabilidades económicas y del cuidado del hogar recaigan sobre ellas. Esta situación ha sido posibilitada por diferentes razones. Por una parte, muchas mujeres han perdido a sus compañeros sentimentales a causa del conflicto armado o como consecuencia de situaciones de guerra, como la Guerra del Oro Verde, y han tenido que ocupar el papel que antiguamente desempeñaban sus maridos como garantes del sustento económico del hogar (ONU Mujeres, 2016) . Por otra parte, está la cultura del gasto del salario del hombre minero, que se podría catalogar como un "mal gasto", puesto que es frecuente que este destine sus recursos económicos al ocio y descuide el núcleo familiar (Rodríguez y Rubiano, 20I6). Lo anterior ocurre comúnmente en hogares de mujeres campesinas y en grupos no minoritarios. Por el contrario, en las comunidades afrocolombianas, en las que la tipología familiar de la mujer minera es matrifocal, estas se desempeñan como jefas del hogar, dado que, como lo destaca Gutiérrez de Pineda (2000), una de las características de las familias de comunidades negras es la ausencia del progenitor, sumado al hecho cultural de que este apenas satisface obligaciones temporales y parciales.

\section{Salud}

El alto grado de informalidad e inestabilidad laboral de las mujeres mineras no les permite acceder a seguridad social ni a cobertura de riesgos laborales, lo que las pone en una situación de desprotección que aumenta los riesgos en su salud. Las largas jornadas laborales, el esfuerzo físico, la exposición a factores ambientales como el sol y la lluvia, la realización de actividades mecánicas y la permanencia en el agua durante tiempos prolongados hacen que las mujeres mineras estén expuestas a riesgos físicos, ergonómicos y biológicos (Donoghue, 2004), lo que aumenta 
su vulnerabilidad a sufrir lesiones como tendinitis, mialgias, artritis, hernia discal o a desarrollar enfermedades como cáncer de piel, infecciones gastrointestinales, entre otros problemas de salud.

En algunos municipios como Zaragoza, en el Bajo Cauca antioqueño, con la llegada de las grandes empresas mineras y de la minería ilegal mecanizada, las mujeres barequeras han sido despojadas de las orillas de los ríos, lo que genera que estas opten por buscar los minerales en los grandes huecos que dejan las retroexcavadoras (Alianza por la Minería Responsable, 2019b) y que aumente el riesgo de accidentes. Un aspecto que afecta de manera diferencial la salud de las mujeres barequeras es el uso del mercurio para separar el oro de las impurezas y la inhalación de sus vapores cuando el material es sometido al fuego para disminuirlas. El mercurio es altamente tóxico y su exposición está asociada con una mayor prevalencia de irregularidad en el ciclo menstrual (RodríguezVillamizar et al., 2015), además de reducir su fertilidad (Rowland et al., 1994). En el caso de mujeres embarazadas, el mercurio puede pasar de la madre al feto en desarrollo y causarle trastornos neurológicos o malformaciones genéticas (García et al., 2017).

\section{Impactos de la minería ilegal en las mujeres mineras}

El cambio en las dinámicas extractivas, como consecuencia de la minería ilegal en muchos territorios del país, reconfigura la vida de los pueblos indígenas y las comunidades afrodescendientes y campesinas que pueden ser irreversibles (Ulloa, 20I6), al transformar los medios de vida, las tradiciones culturales, las relaciones sociales y de género, y la identidad de cada comunidad. Según testimonios de mujeres mineras, los impactos del extractivismo ilegal son en sí mismos una forma de violencia, dado que, si se modifican las condiciones en la extracción minera (las cuales ya son precarias), las mujeres no tienen forma de satisfacer sus necesidades básicas y llevar el sustento a sus hogares (Censat, 20I6). Lo anterior, sumado a la presencia de grandes empresas mineras en sus territorios, constituye la mayor causa de conflictividad ambiental en el país. Según el Atlas de Justicia Ambiental (Environmental Justice Atlas, 2016), Colombia es el país de América Latina con más conflictos ambientales, con setenta y dos casos registrados en 20I6, de los cuales cuarenta y dos fueron por el agua y estuvieron asociados a la explotación minera. A continuación, se explican los principales impactos de la minería ilegal en la vida de las mujeres mineras.

\section{Pérdida de identidad cultural y cambios en las tradiciones}

Sobre este asunto, las mujeres afrodescendientes de La Toma (Cauca) consideran que la minería ilegal mecanizada ha generado condiciones de despojo, destierro y miseria, que modifican la forma de apropiación cultural de los territorios de comunidades negras. En sus palabras manifiestan que "el territorio ha sido nuestro compañero y ha estado con nosotras en momentos de alegrías y tristezas. Nuestras abuelas [...] nos enseñaron que el territorio es la vida y la vida no tiene precio, el territorio es la dignidad y esta no tiene precio” (Censat, 20I6). Estas razones han impulsado a las mujeres a defender la minería artesanal como parte de su identidad territorial (Arias Hurtado, 20I4), pues perder esta tradición conlleva a un fraccionamiento de sus relaciones recreativas, culturales, espirituales, ecológicas y del patrimonio cultural e intangible que este representa (Bermúdez et al., 20I4). 
En las comunidades indígenas, la mujer ha ocupado un papel fundamental en la subsistencia física y cultural de estos pueblos al asociarse con el conocimiento de la agrobiodiversidad, tradición que cambia cuando hay presencia de la minería ilegal mecanizada, pues las mujeres no siembran y prefieren trabajar en las minas. Esto tiene como resultado que se adquieran alimentos ajenos a la dieta tradicional indígena y, con ello, la pérdida de semillas, pues con el descuido de las chagras los cultivos se secan y su recuperación se dificulta (Rodríguez y Rubiano, 2016).

\section{Desplazamiento y pérdida de medios de sustento}

En muchas zonas de tradición minera, históricamente se han presentado fuertes disputas por el territorio, marcadas por la presencia de actores armados e intereses individuales que impulsan el despojo y el desplazamiento de las comunidades. Para las mujeres mineras afrocolombianas esto ha derivado en la pérdida de su autonomía, pues migrar de su colectividad significa cambiar su cultura y buscar nuevas alternativas de sustento. Además, al restringirles el ingreso a los ríos y las vetas de donde obtienen los minerales, se amenazan sus medios de vida (Creer, 2016) y en algunos casos las impulsa a ejercer la prostitución como una forma de obtener ingresos económicos para sus hogares (Rodríguez y Rubiano, 20I6).

\section{Riesgos para la seguridad alimentaria}

Como se mencionó, las mujeres mineras también son agricultoras, actividad que puede ser potencialmente transformada por la minería ilegal mecanizada. Esta puede generar impactos sobre la seguridad alimentaria al deteriorar la calidad del suelo destinado para la agricultura, lo que, como afirman Mina et al. (2015), causa la “esterilidad de las tierras y la devastación generacional de pueblos-tierra-ríos y territorios” (p. 172). Así mismo, la contaminación del recurso hídrico y la monopolización de su uso por la minería ilegal puede afectar la disponibilidad de agua para la agricultura (Bermúdez et al., 20II). Algunas de estas son las razones que han impulsado a la mujer a buscar otras fuentes de ingreso como el chatarreo (Bermúdez et al., 20r4).

\section{Aumento de la violencia hacia la mujer}

En términos de violencia hacia la mujer, uno de los efectos más significativos de la minería ilegal mecanizada es el aumento de las situaciones que impulsan a la mujer a ejercer la prostitución. Sobre este asunto, cabe resaltar que esta es una forma de obtención de ingresos que, según Rodríguez y Rubiano (2016), ha tomado fuerza entre mujeres indígenas en zonas mineras. Sin embargo, esta decisión no siempre es autónoma, por el contrario, las niñas y las mujeres más jóvenes son víctimas de engaños por parte de los mineros foráneos, quienes se aprovechan del hecho de que muchas de ellas nunca han salido de sus comunidades y las engañan con promesas falsas de otorgarles estabilidad laboral, lo que trae como resultado maltratos hacia ellas y la vulneración de sus derechos humanos (Rodríguez y Rubiano, 2016). 


\section{Exclusión de espacios de participación y vulneración de derechos fundamentales}

A pesar de que en Colombia los territorios de los pueblos indígenas y las comunidades étnicas gozan de especial protección, en los dos casos hay presencia de minería ilegal, lo que restringe la participación de las comunidades en el desarrollo de proyectos (que potencialmente pueden afectarlas) y vulnera derechos fundamentales como la consulta previa. Este impacto también se ha presentado en el desarrollo de actividades mineras formales, que han tenido que dirimirse a través de instancias judiciales. Tal es el caso de la acción de tutela interpuesta por Francia Márquez, en la que se aducía la vulneración de los derechos a la vida digna, la consulta previa, al trabajo, al debido proceso y a la autonomía e integridad cultural, como consecuencia de la explotación minera a gran escala en territorios colectivos de comunidades negras. En este caso, la Corte Constitucional de Colombia (20I0), a través de Sentencia T-1045A, reconoció que

esta especial protección implica un proceso mediante el cual el Estado garantiza a las autoridades respectivas y a las comunidades implicadas, directamente la participación y el acceso a la información sobre un programa o plan que se pretenda realizar en su territorio [...], buscando salvaguardar la idiosincrasia de los pueblos indígenas y tribales que habitan el país.

\section{Conclusiones}

En Colombia, la participación de la mujer en la minería es diferenciada y depende de las formas de apropiación cultural del territorio. En comunidades campesinas y grupos no minoritarios, su trabajo puede considerarse como una inclusión desigual, pues las mujeres se limitan a extraer residuos cuando así lo permiten los mineros. En comunidades afrocolombianas, la minería forma parte de la identidad cultural de las mujeres y representa una forma de apropiación ancestral de su territorio; allí, son lideresas y defienden sus derechos humanos a través de formas consolidadas de participación. Las mujeres indígenas, por su parte, se benefician de empleos indirectos de la minería como proveer servicios de alimentación y en algunos casos de prostitución. En todos los grupos, las mujeres mineras no cuentan con estabilidad laboral y son madres cabezas de familia que deben alternar la actividad minera con el cuidado de los hijos y las labores domésticas.

Los derechos humanos de las mujeres mineras se ven afectados principalmente por la presencia de minería ilegal mecanizada, al aumentar el desplazamiento de sus territorios, destruir sus medios de vida, favorecer situaciones de violencia, generar pérdida de su identidad cultural, propiciar la monopolización del recurso hídrico y del suelo, y la exclusión de instancias de participación en las decisiones que puedan afectarlas.

Por estas razones, se recomienda la implementación de políticas con enfoque de género en el sector minero que garanticen la igualdad laboral, así como adelantar procesos de formalización y promover los programas de coexistencia minera entre la tradicional y las medianas y grandes concesiones mineras, con el fin de favorecer el acceso de las mujeres a seguridad laboral, lo que les permitiría estabilidad laboral y proteger sus derechos humanos.

\section{Agradecimientos}

Los avances de la investigación que se muestran en este artículo se nutren del trabajo realizado por el Observatorio Colombiano de Minería (OCMI) de la Universidad del Rosario, en el marco del proyecto "Situación socioeconómica, cultural y ambiental de la mujer en la minería artesanal y a pequeña escala (MAPE) de oro en Colombia”, financiado 
parcialmente por Colciencias, en el marco de la Convocatoria 852 Jóvenes Investigadores e Innovadores 20I8. Los autores agradecen a María Nathalia Muñoz Mosquera y a Sandra Milena Montes Salas, estudiantes de la Facultad de Jurisprudencia de la Universidad del Rosario e integrantes de la línea de investigación de Mujer y Minería del OCMI por su apoyo en la búsqueda de bibliografía para la elaboración de este artículo.

\section{Referencias}

Agencia Nacional de Minería (ANM). (2019). Registro Único de Comercializadores de Minerales. Agencia Nacional de Minería, Gobierno de Colombia. https://tramites.anm.gov.co/Portal/pages/consultaListados/anonimo Listados.jsf

Alianza por la Minería Responsable. (2012). Mujeres en la minería artesanal y de pequeña escala: testimonios de 10 mujeres de Bolivia, Colombia y Perú. ARM. Recuperado el il de abril de 2019 de http://www.responsiblemines.org/wp-content/uploads/2017/0I/MUJERES_EN_LA_MINERIA_AR TESANAL_Y_DE_PEQUENA_ESCALA.pdf

Alianza por la Minería Responsable. (2019a). Historias del territorio: Doris Cuatin. ARM. Recuperado el 23 de mayo de 2019 de http://www.responsiblemines.org/doris-cuatin-coodmilla/

Alianza por la Minería Responsable. (2019b). Historias del territorio: las barequeras de Puerto Jobo. ARM. Recuperado el 23 de mayo de 2019 de http://www.responsiblemines.org/wp-content/uploads/2017/10/Historia-de-comu nidad-mujeres-barequeras.pdf

Alonso, A. A., y Rivera Guzmán, E. (2018). Brechas de género en la minería: la minería artesanal y de pequeña escala colombiana desde una perspectiva de género. ARM. Recuperado el ir de abril de 2019 de http://www.responsiblemines.org/wp-content/uploads/2019/02/DIGITAL-BRECHAS-DE-G\%C3\%8 ${ }_{9} \mathrm{NERO}{ }_{-} \mathrm{M}_{2 \mathrm{I}} \mathrm{C}_{13}$-compressed.pdf

Arias Hurtado, C. (2014). Conflictos territoriales y patrimoniales en el presebre de oro en Colombia. Luna Azul, (39), 207-233. https://doi.org/10.17151/luaz.2014.39.13

Bermúdez, R., Rodríguez, T., y Roa, T. (20II). Mujer y Minería: ámbitos de análisis e impactos de la minería en la vida de la mujeres enfoque de derecho y perspectiva de género. Encuentro Latinoamericano Mujer y Minería. Bogotá, Colombia.

Bermúdez, R., Zambrano, K., y Roa, L. (2014). Los territorios, la minería y nosotras: las mujeres nos preguntamos guía de trabajo. Censat Agua Viva, Amigos de la Tierra Colombia.

Bernasconi, A. (20I4). Afro-Descendant Resistance: A Strategy of Territorial Self-Determination in Colombian Northern Canca. University of Fribourg.

Blanco Acendra, D. (2017). Análisis sociojurídico del barequeo con enfoque étnico. Caso Suárez Cauca. Universidad Nacional de Colombia, Instituto de Estudios Ambientales (IDEA).

Bolívar Rocha, M. C., y Ibarra Melo, M. E. (2017). Las mineras de La Toma. Resistencia negra en defensa de la vida y el territorio. Pensamiento Americano, Io(19), 155-174. https://doi.org/10.21803/pensam.vioir9.35

Carabalí-Viveros, D., y Ladino-Mosquera, V. (20I4). Vida cotidiana de las mujeres mineras de Higuerillos, municipio de Buenos Aires, Cauca. Una mirada a sus familias, su trabajo y su participación política. Prospectiva. Revista de Trabajo Social e Intervención Social, (19), 253-278. https://doi.org/10.25100/prts.voir9.974), 253-278.

Censat. (2016). Marcha de los Turbantes. Movilización Mujeres Afrodescendientes. Censat Agua Viva, Amigos de la TierraColombia. Recuperado el 5 de marzo de 2019 de https://censat.org/es/noticias/marcha-de-los-turbantes-mo vilizacion-mujeres-afrodescendientes-por-el-cuidado-de-la-vida-y-de-los-territorios-ancestrales 
Centro Regional de Empresas y Emprendimientos Responsables de Colombia (Creer). (2016). Evaluación integral sectorial de impactos en derechos humanos: la minería que no se ve. Centro Regional de Empresas y Emprendimientos Responsables.

Chaparro, E., y Lardé, J. (2009). Serie de recursos naturales e infraestructura: El papel de a mujer en la industria minera de Centroamérica y el Caribe. Cepal.

Codelco. (2013). Temas materiales. Reporte sustentabilidad 2013. Codelco. Recuperado el I4 de febrero de 2019 de htt p://w.codelco.cl/temas-materiales/reporte2013/20I4-04-16/192319.html

Comisión Económica para América Latina y el Caribe (Cepal). (2018). ¿Más o menos desiguales?: Una revisión sobre la desigualdad de los ingresos a nivel global, regional y nacional. Revista Cepal, (24), 7-34. https://repo sitorio.cepal.org/bitstream/handle/II362/40024/I/RVEıI8_Amarante.pdf

Congreso de la República. (i de noviembre de 2010). Por medio de la cual se regula la inclusión de la economía del cuidado en el sistema de cuentas nacionales con el objeto de medir la contribución de la mujer al desarrollo económico y social del país y como herramienta fundamental para la definición e implementación de políticas públicas [Ley I4I3 de 2010]. DO: 47.890

Corte Constitucional de Colombia. (2010). Sentencia T-1045A, Expediente T-2761852. Gobierno de Colombia.

Corte Constitucional de Colombia. (2016). Sentencia C-586, Expediente D-II339. Gobierno de Colombia.

Davis, A. (25 de Febrero de 2013). El rol de la mujer negra en la lucha por la equidad de género. (O. Attilio, entrevistador).

Departamento Administrativo Nacional de Estadística (DANE). (2018). Cuenta Satélite de Economía del Cuidado CSEC 20I7. DANE. Recuperado el II de abril de 2019 de https://www.dane.gov.co/files/investigaciones/b oletines/cuentas/ec/Bol_CS_Econo_cuidado_TDCNR_2017.pdf

Departamento Nacional de Planeación (DNP). (2018). Conpes Consejo Nacional de Política Económica y Social República de Colombia . Bogotá: Departamento Nacional de Planeación

Donoghue, A. M. (2004). Occupational health hazards in mining: an overview. Occupational Medicine, 54(5), 283-289. https://doi.org/10.1093/occmed/kqho72

Environmental Justice Atlas. (2016). Environmental Justice Atlas. Enviromental Justice. Recuperado el II de abril de 2019 de https://ejatlas.org/

Extractive Industries Transparency Initiative (EITI). (2016). El estándar del EITI 20I6. Oslo: Extractive Industries Transparency Initiative.

Extractive Industries Transparency Initiative (EITI) Colombia. (2018). Principios. EITI Gobierno de Colombia, Ministerio de Minas y Energía. Recuperado el 27 de mayo de 2019 de http://www.eiticolombia.gov.co/es/ documentos/principios/

Extractive Industries Transparency Initiative (EITI) Secretariat. (2019). Formando un sector extractivo más inclusivo en cuanto a género. EITI. Recuperado el 27 de mayo de 2019 de https://eiti.org/es/blog/formando-sector-extrac tivo-mas-inclusivo-en-cuanto-genero

García, C., Rodríguez, P., y Matínez, E. (2017). El Convenio de Minamata. Así actúa Colombia frente al mercurio. WWF Colombia.

Gobierno de Colombia. (2018). Política en Derechos Humanos del Sector Minero Energético. Ministerio de Minas y Energía. https://www.anm.gov.co/sites/default/files/DocumentosAnm/politica-derechos-humanos-sector -minero-energetico.pdf

González, A. C. (2017). Brechas de género y desigualdad: de los Objetivos de Desarrollo del Milenio a los Objetivos de Desarrollo Sostenible. ONU Mujeres, Fondo de Población de las Naciones Unidas (UNFPA), 
Programa de las Naciones Unidas para el Desarrollo (PNUD). Recuperado el 3 de abril de 2019 de https://colombia.unfpa.org/sites/default/files/pub-pdf/PDF\%2。WEB\% $20 B R E C H A S \% 20 D E \% 20 G E$ NERO\%20Y\%20DESIGUALDAD.pdf

Güiza, L. (2013). La pequeña minería en Colombia: una actividad no tan pequeña. Dyna, 8o(I8I), I09-II7. https://p ure.urosario.edu.co/ws/portalfiles/portal/22062002/35819_181699_I_PB.pdf

Gutiérrez de Pineda, V. (2000). Familia y cultura en Coombia. Universidad de Antioquía.

Jiménez, C., Rojas, P., y Troncoso, R. (20I4). Género, trabajo y subjetividad: el lugar de la mujer en la minería. Persona y Sociedad. Universidad Alberto Hurtado, XXVIII(3), 65-95. https://doi.org/10.11565/pys.v28i3.74

Mina, C., Machado, M., Botero, P., y Escobar, A. (2015). Luchas del buen vivir por las mujeres negras del Alto Cauca. Nómadas(43), 167-183. https://doi.org/10.30578/nomadas.n43aio

Ministerio de Minas y Energía. (2012). Base de datos: Censo Minero Departamental. Gobierno de Colombia, Ministerio de Minas y Energía.

Ministerio de Minas y Energía. (2018). Resoluciónn 40796 de 2018. Por medio de la cual se adopta la Política de Derechos Humanos del Sector Minero Energético. Gobierno de Colombia, Ministerio de Minas y Energía.

Ministerio del Trabajo. (1950). Decreto Ley 3743 de 1950. Gobierno de Colombia, Ministerio del Trabajo.

Moncada, A. (2017). Oro, sexo y poder: violencia contra las mujeres indígenas en los contextos mineros de la frontera Amazónica colombo-venezolana. Textos E Debates, I(3I), 43-53. http://dx.doi.org/10.18227/2217-I448 ted.viizi.4256

Organziación de las Naciones Unidas (ONU). (2015). Transformar nuestro mundo: la Agenda 2030 para el Desarrollo Sostenible. Nueva York: Asamblea General de la Organización de las Naciones Unidas (ONU).

Organziación de las Naciones Unidas (ONU) Mujeres. (2016). Mujeres, las víctimas silenciosas de la esmeralda en Boyacá. En J. D. Restrepo (Dir./Ed.), Mujeres tras el telón de la guerra (pp. 20-35). ONU Mujeres. Recuperado el 22 de mayo de 2018 de https://verdadabierta.com/especiales-v/20I6/mujeres-guerra/descarga/mujeres-tr as-telon-guerra.pdf

Organización para la Cooperación y el Desarrollo Económicos (OCDE). (2019). La OCDE presenta un paquete de herramientas para ayudar a los gobiernos a promover los objetivos de igualdad de género. OCDE. Recuperado el 27 de mayo de 2019 de http://www.oecd.org/centrodemexico/medios/la-ocde-presenta-un-paquete-de-herramient as-para-ayudar-a-los-gobiernos-a-promover-los-objetivos-de-igualdad-de-genero.htm

Posso Quiceno, J. (2010). Las transformaciones del significado y la vivencia de la maternidad, en mujeres negras, indigenas y mestizas del suroccidente colombiano. Sociedad y Economia(18), 59-84. https://www.redalyc.org/ articulo.oa?id=99618003003

Presidencia de la República de Colombia. (5 de agosto de 1959). Código Sustantivo del Trabajo [Decreto Ley 2663 de 1959]. DO: 27.407

Quiroga, C. (2014). Hacia un territorio minero-campesino: propuestas territoriales desde el movimiento socioterritorial en el nordeste de Antioquia, Colombia. En B. Göbel y A. Ulloa (Eds.), Extractivismo minero en Colombia y América Latina (pp. 284-302). Facultad de Ciencias Humanas Universidad Nacional de Colombia.

Ramos, G. (20II). Riqueza inmoral. Revista Semana..

Rodríguez, C. (2013). Contribuciones locales a una historia de la minería en la Amazonía colombiana. Fundación Avina, Tropenbos Internacional Colombia.

Rodríguez, C., y Rubiano, S. (20I6). Las mujeres y la minería ilegal de oro en la Amazonía colombiana. Serie Coleccionable. Estrategia para el Conocimiento y Atención Integral de los Impactos Generados por la Minería en el Departamento del Amazonas, Fondo Patrimonio Natural, IRG, ICAA. 
Rodríguez-Villamizar, L., Jaimes, D., Manquián-Tejos, A., y Sánchez, L. H. (2015). Irregularidad menstrual y exposición a mercurio en la minería artesanal del oro en Colombia. Biomédica Revista del Instituto Nacional de Salud, 35(2), 38-45. https://doi.org/10.7705/biomedica.v35io.2442

Rowland, A., Baird, D., Weinberg, C., Shore, D., Shy, C., y Wilcox, A. (1994). The effect of occupational exposure to mercury vapour on the fertility of female dental assistants. Occupational \&o Environmental Medicine, I(5I), 28-34. https://doi.org/10.1136/oem.5I.I.28.

Soraire, F., Barrionuevo, L., y Wigdor, G. (2013). Mineras. Trabajar y habitar en las minas. Un análisis desde la antropología del trabajar, la producción social del hábitat y la perspectiva crítica de género. Revista de Antropología Experimental, I0(13), I29-I49. https://ri.conicet.gov.ar/bitstream/handle/11336/15157/mineras_tra bajar_y_habitar_en_las_minas.pdf?sequence=I\&isAllowed=y

Soto, Z. (20II). Chile: la mujer en la minería y en la prevención de conflictos mineros. En Taller internacional: Inclusión del enfoque de género en la prevención de conflictos mineros (pp. 43-50). Programa Ciencia y Tecnología para el Desarrollo CYTED. Recuperado el I4 de febrero de 2019 de http://www2.congreso.gob.pe/sicr/cendocbib /con4_uibd.nsf/335BoDiADD4ACEE705257A39005EAD $3 \mathrm{~F} /$ \$FILE/inclusion_del_enfoque_de_genero.pdf

Stefanovic, A., y Saavedra Alvayay, M. (2016). Las mujeres en el sector minero de Chile: propuestas para políticas públicas de igualdad. Cepal. Recuperado el in de abril de 2019 de https://repositorio.cepal.org/bitstream/handle/ıI36 2/40405/4/Si600926_es.pdf

Ulloa, A. (20I4). Geopolíticas del desarrollo y la confrontación extractivista minera: elementos para el análisis en territorios indígenas en América Latina. En B. Göbel y A. Ulloa (Eds.), Extractivismo Minero en Colombia y América Latina (pp. 425-458). Facultad de Ciencias Humanas Universidad Nacional de Colombia.

Ulloa, A. (2016). Feminismos territoriales en América Latina: defensas de la vida frente a los extractivismos. Nómadas, (45), 123-139. https://doi.org/10.30578/nomadas.n45a8

Viezzer, M. (2005). Edición digital: si me permiten hablar:testimonio de Domitila una mujer de las minas de Bolivia. Siglo XXI Editores.

World Economic Forum. (2018). The Global Gender Gap Report. Ginebra: World Economic Forum.

\section{Notes}

* $\quad$ Artículo de investigación

CC BY 